\title{
Errata
}

\section{Erratum. Should Viscous Fiber Supplements Be Considered in Diabetes Control? Results From a Systematic Review and Meta-analysis of Randomized Controlled Trials. Diabetes Care 2019;42:755-766}

https://doi.org/10.2337/dc19-er08a

The authors wish to make a modification in their article as regards a study by Li et al. (2016) to exclude reporting of the short-term, interim effect of $\beta$-glucan on glycemic measures and only retain analysis for the long-term effects, to avoid study duplication. As a result of one comparison exclusion, corrections to the following tables and figures were made: Figures 1-3, Table 1, and relevant Supplementary Data. There are no significant changes in statistical outcomes, with a slight improvement in numerical values in favor of fiber that do not change results interpretation.

The authors confirm that these modifications do not affect the remaining analysis or the conclusion.

The online version of this article (https://doi.org/10.2337/dc18-1126) has been corrected to reflect these changes.
Elena Jovanovski, Rana Khayyat, Andreea Zurbau, Allison Komishon, Nourah Mazhar, John L. Sievenpiper, Sonia Blanco Mejia, Hoang Vi Thanh Ho, Dandan Li, Alexandra L. Jenkins, Lea Duvnjak, and Vladimir Vuksan 